\title{
A STUDY ON WIND SPEED DISTRIBUTIONS
}

\author{
Indhumathy. $\mathbf{D}^{\mathbf{1}}$, Seshaiah.C. $\mathbf{V}^{\mathbf{2}}$, Sukkiramathi.K ${ }^{\mathbf{3}}$ \\ ${ }^{1}$ Assistant Professor, Department of Mathematics, Sri Ramakrishna Engineering College, Coimbatore, Tamilnadu, India \\ ${ }^{2}$ Professor, Department of Mathematics, Sri Ramakrishna Engineering College, Coimbatore, Tamilnadu, India \\ ${ }^{3}$ Assistant Professor, Department of Mathematics, Sri Ramakrishna Engineering College, Coimbatore, Tamilnadu, India
}

\begin{abstract}
Owing to the growing environmental concern harnessing renewable energy sources became absolutely necessary like solar, wind etc. Wind energy is more sensitive to variations with topography and wind patterns compared to solar energy. The wind energy distribution is the basis for the assessment of wind energy potential needed for the design of wind farms. Accurate wind speed modeling is critical in estimating wind energy potential for harvesting wind power effectively. The quality of wind speed assessment depends on the capability of chosen pdf to describe the measured wind speed frequency. The objective of this study is to describe (model) wind speed characteristics using proposed generalized skew logistic distribution. In order to decide the most suitable site for installing a wind farm as well as to select a fitting wind turbine model it is necessary to carry out a careful wind energy resource evaluation. In this study we propose the generalized skew logistic distribution for the description of wind speed distribution. This distribution is flexible enough to accommodate the shape of wind speed data and include some well known distribution as special cases, also we evaluate the performance of this distribution by using wind speed data at Mupandal (1) at kanya kumari in India. Results show that this generalized skew logistic distribution is a better fit followed by WW pdf.
\end{abstract}

Keywords: skew $t$, skew logistic, likelihood method, and statistical methods...

\section{INTRODUCTION}

In recent years it has been investigated that the fitting of specific distribution to wind speed is required for use in practical application as air pollution modeling, estimation of wind loads on building and wind power analysis. So a model is required for wind speed distribution. Extensive literature search indicates that various parametric distribution models have been presented to estimate wind speed distributions, which are used in a variety of applications such as wind farm planning, long term strategy of wind generators, and reliability evaluation of wind resources. These parametric distribution models include Weibull, Rayleigh, Lognormal, inverse Gaussian , Gamma, Erlang, Burr, Skew t , Skew logistic, Mixture distributions etc,. In this paper wind speed analysis is done using the generalized skew logistic distributions and the performance of the same are compared with several distributions. The study reveals that generalized skew logistic distribution gave a good fit followed by the WW pdf. These results are supported by the statistical tests and graph.

Celik [1] made statistical analysis and summarized that Weibull model was better than Rayleigh model. Akdag.S.A [5] discussed about the two component Weibull distribution and stated that WW gave a goodfit. Tian Pau,C [16] used Rayleigh, Weibull and gamma distribution and its generalized form. Gupta and Kundu [3] discussed about the generalized logistic distribution. Several distributions were discussed in literature to find the best fit.
In this discussion we have proposed generalized skew logistic distribution. We have analysed several distributions for the recorded wind data at Mupandal (1) at Kanya kumari in India for5 years and compared the fits of those with our proposed generalized skew logistic distribution. We have fitted all these generalized skew logistic distribution to the measured wind speed data by the method of likelihood method

\section{WIND SPEED DISTRIBUTIONS}

In literature, the pdf is defined as a mathematical function describing the relative likely hood for this random variable to occur at a given point in the observation space. This section of the paper, presents the different pdf to the wind speed model.

\subsection{Skew Logistic Distribution}

The pdf of the usual skew logistic distribution is given by

$$
\begin{gathered}
f_{S L}(v ; \lambda)=2 h(v) H(\lambda v),-\infty<v<\infty \\
=\frac{2 \exp (-v)}{(1+\exp (-v))^{2}(1+\exp (-\lambda v))}
\end{gathered}
$$

Where $\lambda \in R$ is the skewness parameter $h(v)$ is the standard normal parameter and $H(v)$ is the standard logistic cdf. 


\subsection{Generalised Logistic Distribution}

The pdf of the generalized logistic distribution is given by

$$
g_{\propto}(v)=\frac{1}{B(\propto, \propto)} \frac{\exp (-\infty v)}{(1+\exp (-v))^{2 x}},-\infty<v<\infty \text { and } \infty>0-(2)
$$

The distribution has cdf

$$
G_{\propto}(v)=\frac{B_{y}\left(\alpha_{c} \propto x\right]}{B\left(\alpha_{i} \propto\right)} \text { where } y=(1+\exp (-v))^{-1}
$$

And $B(\propto, \propto)=\frac{[r(\propto)]^{2}}{\Gamma(2 \propto)}$

\subsection{Generalised Skew Logistic Distribution}

The pdf of the generalized logistic distribution is given by

$$
f_{G S L D}(v ; \propto, \lambda)=2 g_{\propto}(v) G_{\propto}(v), \lambda \in R
$$

$$
\text { And }-\infty<v<\infty, \infty>0
$$

The pdf of the generalized logistic distribution with the location parameter $\mu \in R$ and scale parameter $\sigma>0$ is given by

$$
\begin{gathered}
f_{G S L D}(v ; \mu, \sigma, \propto, \lambda)=\frac{2}{\sigma} g_{\propto}\left(\frac{v-\mu}{\sigma}\right) G_{\propto}\left(\lambda\left(\frac{v-\mu}{\sigma}\right)\right) \\
-\infty<v<\infty, \infty>0, \lambda \in R
\end{gathered}
$$

\subsection{Skewed Generalised Error Distribution}

The skewed generalized Error distribution (SGED) suggested by [11] and its pdf and cdf are defined by equations (6) and (7) respectively.

$$
\begin{aligned}
& f_{S G E D}(v)=\frac{\theta_{2} e^{-\left(\frac{\left\|v-\theta_{0}\right\|}{\left(\theta_{1}\left(1+\operatorname{sign}\left(v-\theta_{0}\right) \theta_{g}\right)\right.}\right)^{\theta_{2}}}}{2 \theta_{1} \Gamma\left(\frac{1}{\theta_{2}}\right)} \\
& F_{S G E D}(v)=\int_{-\infty}^{v} \frac{\theta_{2} e^{-\left(\frac{\left\|t-\theta_{0}\right\|}{\left(\theta_{1}\left(1+\operatorname{sign}\left(t-\theta_{0}\right) \theta_{g}\right)\right.}\right)^{\theta_{2}}}}{2 \theta_{1} \Gamma\left(\frac{1}{\theta_{2}}\right)} d t
\end{aligned}
$$

Where $\Gamma$ denotes the gamma function and $\theta_{2}$ is the location $\theta_{1}$ is the scale, $\theta_{3}$ are shape parameters. Also the SGED generates some distributions such as normal, skewed normal and Laplace in special cases.

\subsection{Skewed t Distribution}

The Skewed t distribution (STD) was suggested by [2] and the pdf and cdf are respectively given as follows

$$
f_{S T D}(v)=\frac{2}{2 \theta_{1} \theta_{2}^{1 / 2} \beta\left(1 / 2^{2}, \theta_{2}\right)\left(1+\frac{\left|v-\theta_{0}\right|^{2}}{\theta_{2}\left(1+\operatorname{sign}\left(v-\theta_{0}\right) \theta_{3}\right)^{2} \theta_{1}^{2}}\right)^{1 / 2+\theta_{2}}}
$$

And

$$
F_{S T D}(v)=\int_{-\infty}^{v} \frac{2}{2 \theta_{1} \theta_{2}{ }^{1 / 2} \beta\left(1 / 2, \theta_{2}\right)\left(1+\frac{\left|t-\theta_{0}\right|^{2}}{\theta_{2}\left(1+\operatorname{sign}\left(t-\theta_{0}\right) \theta_{3}\right)^{2} \theta_{1}^{2}}\right)^{1 / 2+\theta_{2}}} d t
$$

Where $\theta_{0}$ and $\theta_{1}$ location and scale parameters respectively are $\theta_{2}$ and $\theta_{3}$ are shape parameters in $\mathrm{m} / \mathrm{s}$

\subsection{Weibull - Weibull Distribution}

The PDF which depends on five parameters $\left(\mathrm{v} ; k_{1}, c_{1}, k_{2}, c_{2}, w\right)$ is given by

$$
f f\left(v, k_{1}, c_{1}, k_{2}, c_{2}, w\right)=w f\left(v, k_{1}, c_{1}\right)+(1-w) f\left(v, k_{2}, c_{2}\right)(10)
$$

where $c_{1}$ and $c_{2}$ are shape parameters

and $k_{1}$ and $k_{2}$ are shape parameters

\section{WIND SPEED DATAS}

The measured wind speed datas for the month of August 2010 are given in the Table- 1and graphical representation in Fig-1

Table 1 wind speed datas

\begin{tabular}{|c|c|c|c|c|c|}
\hline Day & $\begin{array}{c}\text { Wind } \\
\text { speed(k/h) }\end{array}$ & Day & $\begin{array}{c}\text { Wind } \\
\text { speed(k/h) }\end{array}$ & Day & $\begin{array}{c}\text { Wind } \\
\text { speed(k/h) }\end{array}$ \\
\hline $\mathbf{1}$ & 11 & $\mathbf{1 2}$ & 8 & $\mathbf{2 3}$ & 14 \\
\hline $\mathbf{2}$ & 10 & $\mathbf{1 3}$ & 14 & $\mathbf{2 4}$ & 16 \\
\hline $\mathbf{3}$ & 13 & $\mathbf{1 4}$ & 13 & $\mathbf{2 5}$ & 13 \\
\hline $\mathbf{4}$ & 6 & $\mathbf{1 5}$ & 8 & $\mathbf{2 6}$ & 11 \\
\hline $\mathbf{5}$ & 11 & $\mathbf{1 6}$ & 11 & $\mathbf{2 7}$ & 10 \\
\hline $\mathbf{6}$ & 13 & $\mathbf{1 7}$ & 11 & $\mathbf{2 8}$ & 5 \\
\hline $\mathbf{7}$ & 8 & $\mathbf{1 8}$ & 14 & $\mathbf{2 9}$ & 10 \\
\hline $\mathbf{8}$ & 6 & $\mathbf{1 9}$ & 13 & $\mathbf{3 0}$ & 14 \\
\hline $\mathbf{9}$ & 8 & $\mathbf{2 0}$ & 13 & $\mathbf{3 1}$ & 11 \\
\hline $\mathbf{1 0}$ & 14 & $\mathbf{2 1}$ & 8 & & \\
\hline $\mathbf{1 1}$ & 16 & $\mathbf{2 2}$ & 18 & & \\
\hline
\end{tabular}




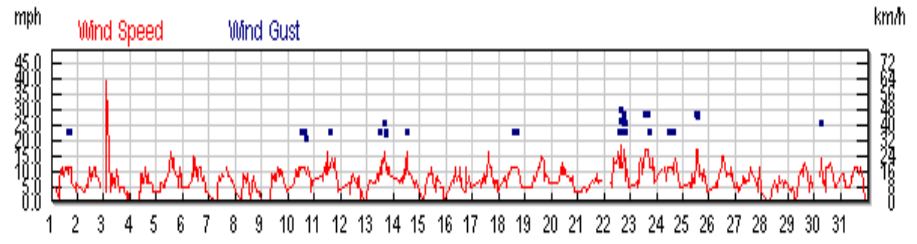

Fig 1: Representation of monthly wind speed (Aug 2010)

\subsection{Estimation of Parameters}

The estimation of parameters of all the pdfs were carried out using likelihood method and there listed in the following table.

Table 2 Computed parameter values of different pdfs

\begin{tabular}{|c|c|c|c|c|c|}
\hline PDF & Parameters & Values & PDF & Parameters & Values \\
\hline \multirow[t]{3}{*}{ SL } & $\mu$ & 2.328 & \multirow[t]{3}{*}{ GL } & $\mu$ & 3.048 \\
\hline & $\sigma$ & 0.550 & & $\sigma$ & 0.930 \\
\hline & $\lambda$ & 3.713 & & $\lambda$ & 5.041 \\
\hline \multirow[t]{4}{*}{ ST } & $\mu$ & 1.924 & \multirow[t]{4}{*}{ SGE } & $\theta_{0}$ & 1.3423 \\
\hline & $\sigma$ & .9440 & & $\theta_{1}$ & 0.9245 \\
\hline & $\lambda$ & 4.3972 & & $\theta_{2}$ & 1.5024 \\
\hline & $\alpha$ & 26501.23 & & $\theta_{3}$ & 0.7523 \\
\hline \multirow[t]{5}{*}{$\mathbf{W W}$} & $\mathrm{w}$ & 0.5197 & \multirow[t]{5}{*}{ GSL } & $\mu$ & 2.271 \\
\hline & $k_{1}$ & 3.1775 & & $\sigma$ & 195.80 \\
\hline & $k_{2}$ & 10.0174 & & $\lambda$ & 4.418 \\
\hline & $c_{1}$ & 1.8689 & & $\propto$ & 76605.63 \\
\hline & $c_{2}$ & 4.1004 & & & \\
\hline
\end{tabular}

\section{GOODNESS-OF-FIT CRITERIA}

To compare a theoretical pdf with the measured wind data three kinds of statistical tests are considered

\section{1 $\mathrm{R}^{2}$ Error}

$R^{2}$ test is widely used for goodness of fit. A larger value of $R^{2}$ indicates a better fit. $R^{2}$ is defined by

$$
R^{2}=\frac{\sum_{i=1}^{n}\left(\hat{F}_{i}-\bar{F}\right)^{2}}{\sum_{i=1}^{n}\left(\hat{F}_{1}-\bar{F}\right)^{2}+\sum_{i=1}^{n}\left(F_{i}-\hat{F}_{i}\right)^{2}}
$$

\subsection{The Max Error}

The max error estimates the error calculated by distribution function and observed wind speed data respectively. The smaller the errors, the better the fit is . This is defined by

$$
\text { Max Error }=\max |C(v)-O(v)|
$$

\subsection{Chi- Square Error}

Chi-Square error is used to asses whether the observed probability differs from the predicted probability. Chi-Square error is defined by

$$
\chi^{2}=\sum_{i=1}^{n} \frac{\left(F_{i}-\widehat{F}_{i}\right)^{2}}{F_{i}}
$$

Table- 3; Statistical Errors for different distributions

\begin{tabular}{|l|l|l|l|}
\hline PDF & K-S Error & $R^{2}$ Error & $\chi 2$ \\
\hline WW & $\mathbf{0 . 0 7 4}$ & $\mathbf{0 . 9 9 9 3}$ & $\mathbf{0 . 2 6 8 1}$ \\
\hline SLD & $\mathbf{0 . 0 8 4}$ & $\mathbf{0 . 9 8 2 7}$ & $\mathbf{0 . 8 3 4 5}$ \\
\hline STD & $\mathbf{0 . 0 7 7}$ & $\mathbf{0 . 9 8 5 2}$ & $\mathbf{0 . 2 6 8 4}$ \\
\hline GLD & $\mathbf{0 . 0 9 7}$ & $\mathbf{0 . 9 6 4 2}$ & $\mathbf{1 . 1 0 5 5}$ \\
\hline GSLD & $\mathbf{0 . 0 7 3}$ & $\mathbf{0 . 9 9 9 4}$ & $\mathbf{0 . 2 6 8 0}$ \\
\hline SGED & $\mathbf{0 . 0 8 4}$ & $\mathbf{0 . 9 8 4 6}$ & $\mathbf{0 . 8 2 1 6}$ \\
\hline
\end{tabular}

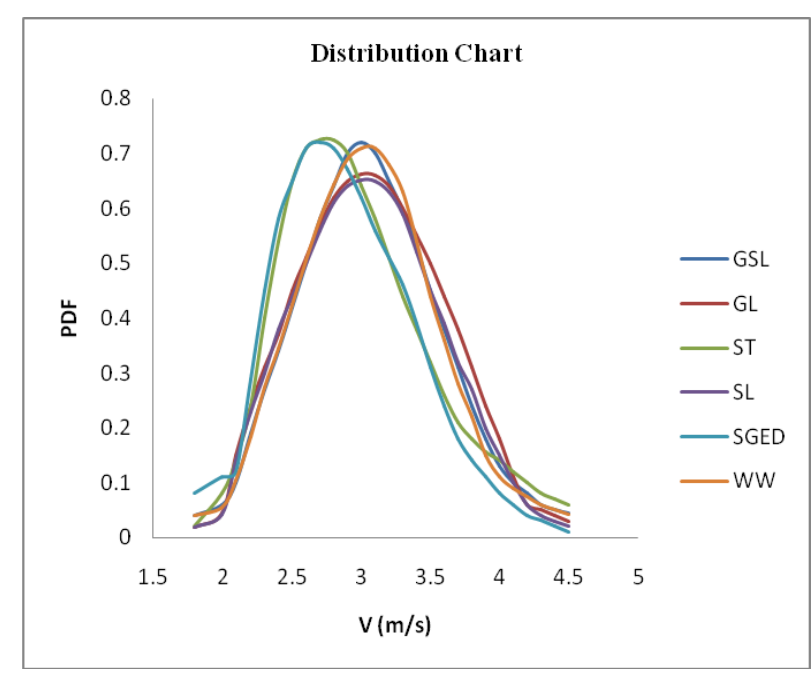

Fig: $\mathbf{2}-$ Graphical representation

\section{CONCLUSIONS}

Wind speed data at kanya kumari were used in evaluating different PDf $s$ to access their suitability.Wind speed data for station 1(Mupandal) recorded at a height of $25 \mathrm{~m}$ at Latitude $\mathrm{N}$ at $8^{\circ} 15^{\prime \prime} 30^{\prime}$, Longitude $\mathrm{E}$ at $77^{\circ} 33^{\prime \prime} 20^{\prime}$ are used for wind speed analysis. Wind data for a period of 5 years is used

The parameters were estimated using the likelihood method carried out in MATLAB software. Computed parameters were used to find the different PDF's. The generalized skew logistic distribution showed a goodfit followed by the WW pdf and the result is evident from the statistical errors in Table 2. On the whole GSLD followed by WW pdf fits and this result is supported by the fig 1 also 


\section{REFERENCES}

[1]. Celik ,A .N ., " A statistical analysis of wind power density based on the Weibull and Rayleigh models at the southern region of Turkey “, Renew Energy ,Vol 29, pp. 593604,2003 .

[2]. Arellano Valle,R.B and Azzalini, A "The centered parameterization and related quantities of the skew $t$ distribution" , Journal of Multivariate Analysis,113,7390,2013.

[3]. Gupta, R.D and Kundu , D., "Generalized logistic distribution", Journal of Applied Statistical Sciences,18,5166, 2010.

[4]. Nadarajah, S.," The skew logistic distribution", A St A Advances in Statistical Analysis,93,187-203, 2009.

[5]. Akdag S. A, Bagiorgas , H.S, " Use of two component Weibull mixtures in the analysis of wind speed in the Eastern Mediterranean “, Appl Energy, 87,2566-73, 2010.

[6]. Chang ,T .P.," Estimation of wind Energy potential using different probability density functions “,Appl Energy , Vol 88, pp. 1848-56, 2011

[7]. Surucu ,B . A ., "Power comparison and stimulation study of goodness-of-fit tests “, Comput math Appl, Vol 56 , pp $.1617-25,2008$

[8]. Chang ,T .P.," Performance comparison of six numerical methods in estimating Weibull parameters for wind Energy Application “, Appl Energy, Vol 88, pp. 272-82,2011.

[9]. Akpinar, S., Akpinar, .E.K . "Wind energy analysis based on maximum entropy principle(MEP)- type distribution function". Energy Convers Manage , 48 1140-09, 2007.

[10]. MC Donald .B,. " Model selection : some generalized distribution " Commun Stat Theory Methods, 16 (4), 104974, 1987.

[11]. Theodossiou . P,. "Skewed generalized Error distribution of financial assests and option pricing ,2002.

[12]. Gombay,E., Harrath., L. ," an application of the likekyhood method to change point detection", Envirometrics, 8, 459-467, 1997.

[13]. Dorvlo A.S., " Estimating wind speed distribution". Energy convers manag, 43(17) ,2311-18, 2002.

[14]. Sfetsons. A,. "A novel approach for the forecasting of mean hourly wind speed time series". Renew Energy ,27(2), 163-74, 2002.

[15]. Tian Pau,C, " Estimation of wind energy potential using different probability density functions" . Applied energy ,88(5) , 1848-1856, 2011.

[16]. Garcia. A, Torres .J.L, Prieto , E., De Franciso A,. “ Fitting wind speed distributions: a case study", Solar Energy ,62,139-144, 1998. 